\title{
La planificación forestal participada como base de la gobernanza rural
}

\author{
Public forestry planning as the basis of rural governance
}

\author{
Xabier Bruña-García a, Manuel Marey-Pérez ${ }^{\text {a* }}$

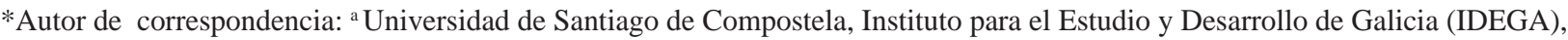 \\ Departamento de Ingeniería Agroforestal, Lugo, España, tel.: + 34 982823248, ext. 23248 / 23247, manuel.marey@usc.es
}

\section{SUMMARY}

Social participation in decision-making on land-use management and planning is essential to achieve viable and lasting solutions. Particularly, a joint action by all sectors involved in the development of a tactical and strategic program of natural resources planning, allowing for a consensus final document in which solutions are formulated to the future management of the resources of the region, is being planned. In rural areas, the search for consensus among stakeholders is the most important factor in planning the territory to be the common denominator in all the activities taking place in it. Within the public participation process and the achievement of consensus, it is necessary to have a balanced and representative portion of the various stakeholders in the area. This article discusses the public participation process conducted within the program of forest planning of the A Fonsagrada-Os Ancares district in order to validate this methodology for application in future participatory processes in rural areas.

Key words: forest plan, planning, public participation, rural governance, rural area.

\section{RESUMEN}

La participación de la sociedad en la planificación y ordenación del territorio es necesaria para conseguir soluciones duraderas y viables a lo largo del tiempo. En concreto, la actuación en conjunto de todas las partes involucradas en la creación de un plan forestal, de carácter táctico y estratégico, permite la obtención de un documento consensuado socialmente que permite obtener soluciones armónicas a la futura gestión de recursos rurales de la región. Así, buscar el consenso entre los diferentes grupos de interés de un área rural es un factor decisivo en planificación rural puesto que, el territorio es el denominador común a todas las actividades en él desarrolladas. Por tanto, dentro del proceso participado, y para conseguir la gobernanza rural, es imprescindible tener una muestra representativa y equilibrada de los stakeholders presentes en el área. El presente artículo evalúa el desarrollo de un proceso participado dentro del plan del distrito forestal de A Fonsagrada- Os Ancares (Galicia, noroeste de España) para validar dicha metodología y su posible aplicación en futuros procesos participados en otros territorios rurales.

Palabras clave: gobernanza rural, participación pública, plan forestal, planificación, zona rural.

\section{INTRODUCCIÓN}

Los recursos naturales suelen tener conflictos complejos que se asocian habitualmente a problemas originados en los distintos puntos de partida de la realidad por parte de las personas implicadas (Gritten et al. 2009). En relación con esto, y como solución holística a esta problemática de base, la gestión y la planificación de los recursos rurales se fundamentan, cada vez con mayor peso, en los denominados enfoques multidisciplinares de evaluación integral, que analizan los impactos social, ambiental y económico de la ordenación desarrollada en la zona objeto.

Dentro de los recursos rurales de una región, el monte (entendido en un sentido amplio e incluyendo además de la parte arbórea, la parte arbustiva y herbácea) es fuente de múltiples recursos y servicios pero, es también origen de numerosos y diversos conflictos sociales. En su estudio sobre conflictos medioambientales en materia forestal para diferentes regiones europeas y norteamericanas, Hellström (2001) concluye que encajar los diferentes intereses e integrar los diferentes colectivos demandantes de bienes y servicios forestales, determinaron un aumento significativo del número de conflictos sociales a partir de los años 1960 y 1970, conflictos de diferente forma y nivel de intensidad.

Para resolver los conflictos que existen en un territorio y lograr un consenso equilibrado entre los stakeholders implicados, la principal vía de trabajo en la actualidad es integrar participación pública en el proceso planificador. En su definición inicial, participación pública fue considerada una herramienta de concienciación mediante la que las personas inician la comprensión de sus posiciones políticas y de la necesidad de contribuir a la sociedad (Utton et al. 1976). Posteriormente, la participación pública pasó a ser un principio básico para aportar transparencia e intercambiar información, es decir, un requisito fundamental para construir consenso. Actualmente, participación públi- 
ca se entiende como un proceso voluntario en el que las personas (de forma individual o a través de grupos organizados), intercambian información, expresan sus opiniones y articulan intereses, fomentando su potencial para decidir en el resultado (ILO 2000).

Además, en todo proceso para tomar decisiones dentro de una planificación territorial pueden darse distintos niveles de participación pública, desde la participación pasiva, donde los interesados son informados sobre las decisiones tomadas por otros, hasta la participación interactiva, donde se requiere decisiones conjuntas compartiendo responsabilidades (Higgs et al. 2008).

En la actualidad está aceptado que a través de los métodos participativos se puede conseguir una gestión sustentable de los recursos rurales de un territorio $\mathrm{y}$, cada vez más, los habitantes locales exigen más influencia y voz en cómo se gestionarán y planificarán dichos recursos (Leskinen 2004). Este hecho requiere que la planificación y gestión de los recursos rurales se asienten en la participación directa y activa de un número mayor de interesados, así como en la agregación de las percepciones, visiones e intereses que la población tenga de dichos recursos, especialmente en áreas rurales.

La participación social no es algo nuevo en la planificación, pero su presencia a nivel forestal es reciente, constituyendo una práctica poco desarrollada desde un ámbito local (Leskinen 2004). No obstante, cada vez más habitantes están interesados en tener más peso y control en las decisiones vinculadas con la gestión y planificación forestal (Buchy y Hoverman 2000). Paralelamente, los profesionales rurales necesitan, cada vez con más intensidad, comunicar sus acciones e ideas con diversos profesionales, así como con el amplio abanico de partes no profesionales interesadas en el mundo rural. Teniendo en cuenta a Sugimura y Howard (2008), todas las personas interesadas en un proceso de planificación forestal deben participar en su desarrollo, especialmente, en sus últimas etapas, siendo la participación pública una herramienta fundamental para alcanzar la ordenación sostenible (ILO 2000).

Tradicionalmente, la planificación del ámbito forestal se ha caracterizado y se caracteriza por ser sectorial, vertical y dirigida (Bruña-García y Marey-Pérez 2014). Frente a esta situación, alcanzar la gobernanza forestal requiere, sin embargo, implicar a todos los actores del territorio desde el principio del proceso planificador. Para alcanzar este objetivo, uno de los caminos que se está desarrollando fuertemente en el campo forestal es la participación pública. Así, autores como Sheppard y Meitner (2005), Pykäläinen et al. (2007) y Hiltunen et al. (2009), entre otros, han desarrollado diversos procesos participados en el ámbito forestal. En dichos estudios, la población de trabajo eran sociedades estructuradas con experiencias previas en participación pública, pero sin integración en materia de planificación forestal.

En zonas rurales que carecen de experiencia previa con participación pública, uno de los obstáculos de la participación social es la carencia de formación e información de los distintos agentes o colectivos implicados en la planificación y gestión de los recursos. Por esta causa, la fase previa de difusión es fundamental para alcanzar el éxito en esta última, puesto que, una correcta difusión permitirá que la población esté adecuadamente informada y se despierte su interés por participar de forma activa en todo el proceso de planificación desde sus inicios.

En España, la ley básica de montes (Ley 43/2003, modificada posteriormente por la Ley 10/2006) exige que toda planificación forestal esté acompañada por la participación y consulta públicas a las partes implicadas o interesadas, proceso relativamente compatible o similar con la ordenación de recursos naturales. Esta exigencia de un proceso participado en el ámbito forestal se vio reforzado con la posterior aprobación de un nuevo texto normativo estatal (Ley 27/2006), regulándose los derechos para participar y para acceder a la justicia e información en materia ambiental, así como los pilares elementales de cualquier proceso participado para las distintas etapas de aprobación y desarrollo de cualquier plan de ordenación o planificación.

El presente artículo tiene como objetivo presentar una metodología de participación pública integrada en la planificación del recurso forestal de un territorio concreto. La validación de dicha metodología se realiza como etapa integrante en un plan táctico forestal, de nivel subregional, desarrollado en A Fonsagrada- Os Ancares, distrito forestal perteneciente a la región de Galicia, situada al Noroeste de la Península Ibérica.

\section{MÉTODOS}

El proceso participado propuesto se integró en el desarrollo del plan forestal del distrito A Fonsagrada - Os Ancares, región eminentemente rural que carece de experiencias previas en participación pública (figura 1). Situado en la zona oriental de Galicia (noroeste de España), este distrito forestal está formado por nueve municipios, para una superficie conjunta de $1.728 \mathrm{~km}^{2}$ y una población total de 19.111 habitantes en el año 2008 (Marey-Pérez 2009). Con un reparto poblacional muy desigual entre municipios, la densidad de población en la zona es de 11,06 habitantes $\mathrm{km}^{-2}$, cifra notablemente inferior a la media europea (116 habitantes $\mathrm{km}^{-2}$ ), revelando el carácter indiscutiblemente rural de la región.

El distrito A Fonsagrada- Os Ancares destaca por la existencia de numerosos propietarios forestales privados individuales (Rodríguez-Vicente y Marey-Pérez 2010) y explotaciones agrarias (Álvarez-López et al. 2008), gestores de un mosaico de tierras de cultivo agrícola y masas forestales (Díaz-Varela et al. 2009). En los últimos 50 años, el área de estudio ha perdido el 60 \% de su población (Marey-Pérez 2009), hecho que ha favorecido el incremento de su superficie forestal hasta alcanzar el $80 \%$ de toda la superficie (Marey-Pérez 2009). Desde el año 2004 , el 40,3\% del distrito está bajo algún régimen de protección medioambiental (figura 2), siendo previsible que 


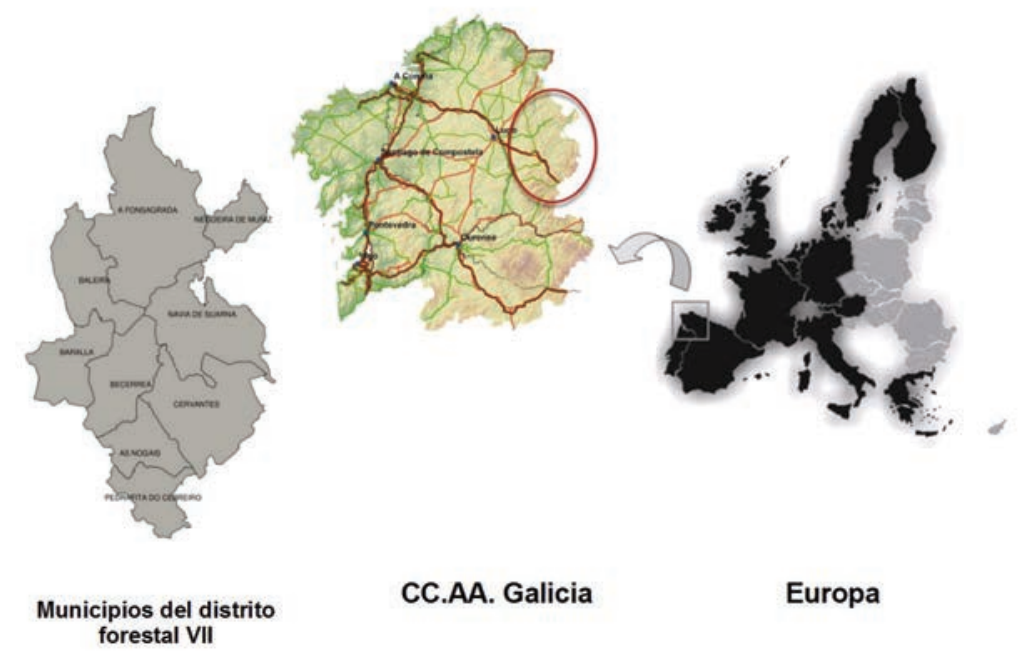

Figura 1. Situación del área de estudio, distrito forestal A Fonsagrada- Os Ancares (Galicia, noroeste de España).

Situation of the study area, A Fonsagrada- Os Ancares forestry district (Galicia, northwest of Spain).

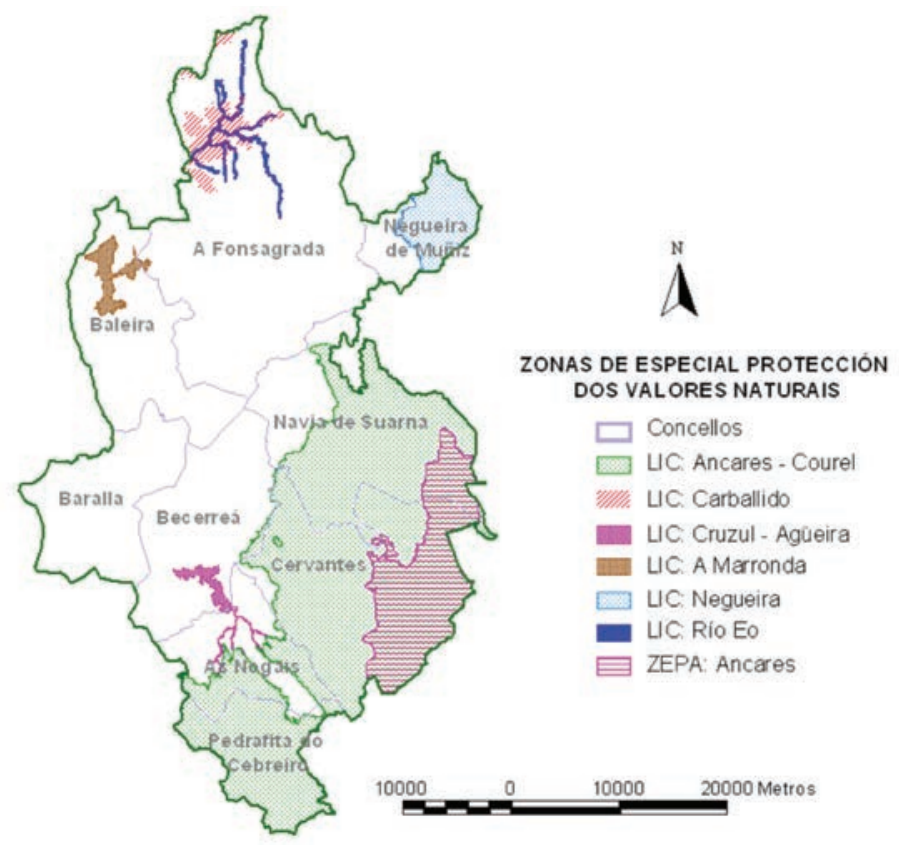

Figura 2. Espacios protegidos del área de estudio, distrito forestal A Fonsagrada- Os Ancares.

Protected forests in the study area, A Fonsagrada- Os Ancares forestry district.

dicha cifra aumente en los próximos años (Marey-Pérez y Rodríguez-Vicente 2011).

El principal problema ambiental y, particularmente forestal, del distrito son los incendios forestales (FuentesSantos et al. 2013). Entre 1991 y 2008 se registraron 4.764 incendios forestales en la zona, arrasando una superficie de 19.777 hectáreas (Marey-Pérez 2009). La mayoría de estos incendios fueron causados por la actividad humana, siendo el $87 \%$ incendios intencionados que quemaron un total de 15.398,56 hectáreas en el distrito (Fuentes-Santos et al. 2013).

Reflejando el carácter rural de la zona de estudio, se indica que el peso del sector industrial es mínimo en su socioeconomía, dado que las pocas empresas existentes constan de uno a nueve empleados, existiendo solamente una industria con más de 50 trabajadores en el municipio 
de A Fonsagrada. Con un 45,79 \% de su población activa, las actividades relacionadas con el sector primario (agricultura, ganadería y selvicultura) tienen, sin embargo, una importancia significativa en el distrito, claramente superior a la media de Galicia (7,66 \%). Finalmente, el sector secundario de A Fonsagrada- Os Ancares es el menos relevante en la zona, ocupando menos del 5,90 \% de su población.

En la región, las experiencias en participación pública son prácticamente inexistentes, concentrándose en etapas de información pública propias de procesos administrativos; además de alguna reunión esporádica centrada en la previsible declaración de un parque natural. A estos precedentes, hay que tener en cuenta la desconfianza de sus habitantes. Ambos factores aumentan claramente los condicionantes para definir procesos participativos adecuados en este territorio.

La participación del plan del distrito forestal de A Fonsagrada- Os Ancares se articuló en tres fases claramente diferenciadas: divulgación, debate y toma decisiones, y resultados (figura 3). El trabajo se desarrolló por un equipo multidisciplinar de 18 personas que dedicaron un total de 561 horas de trabajo entre diciembre de 2008 y junio de 2009. Los resultados obtenidos en dicho proceso se incorporaron posteriormente en la planificación forestal de la región, esta última diferenciada en tres fases: participación pública, evaluación ambiental y redacción técnica.

Fase de divulgación. Esta fase comenzó con la clasificación y caracterización de los agentes implicados en la gestión forestal de la región, estableciendo a continuación la estrategia adecuada a cada colectivo. Las acciones desarrolladas fueron, entre otras, el análisis de los stakeholders, la creación de la estrategia general (reuniones para informar en cada municipio y realizar difusión local) y el desarrollo de la estrategia específica (notificaciones y reuniones con los principales stakeholders).
Por un lado, la estrategia general consistió en la celebración de reuniones en cada municipio del distrito forestal de estudio, es decir, nueve reuniones iniciales, a las que se sumaron dos más de refuerzo. La divulgación o convocatoria de estas reuniones consistió en la colocación de 466 carteles (figura 4), colocados en 164 parroquias (representan el $99 \%$ de la totalidad de parroquias del distrito) y 425 núcleos habitados, lo que representa el $43 \%$ del total (figura 5). Esta etapa se reforzó además con la difusión de 1.600 trípticos en aquellos lugares más accesibles (centros médicos, ayuntamientos y oficinas administrativas, entre otros).

Por otro lado, dentro de la estrategia específica, se mantuvo primeramente una reunión con alcaldes y corporaciones locales (administración local) de cada municipio del distrito forestal para informar del inicio del plan forestal y solicitar su cooperación durante el proceso de participación. Posteriormente, se enviaron 329 cartas informativas del proceso, con acuses de recibo, destinadas a montes vecinales (comuneros), asociaciones y diversos colectivos de interés (clave) en la gestión territorial del distrito.

Finalizadas las etapas anteriores de la fase de difusión, se inició la inscripción de interesados en el proceso participativo del plan forestal. Para esta fase se estableció un plazo de tres semanas desde la finalización de las reuniones informativas anteriores (Abelleira-Yáñez et al. 2011).

Para alcanzar la máxima divulgación del inicio del plan forestal y su apertura general a toda la sociedad, de forma transparente y democrática, se abrió un sitio web (www. planforestaldistritovii.com), que funcionó entre diciembre del 2008 y agosto del 2009. Durante su operatividad, dicho portal contó con un total de 2.271 visitas (lo que supone 9,16 visitas por día) y 1.030 usuarios diferentes (en torno al $5 \%$ de los habitantes del distrito forestal), siendo el tiempo promedio de visita de cinco minutos y 26 segundos.

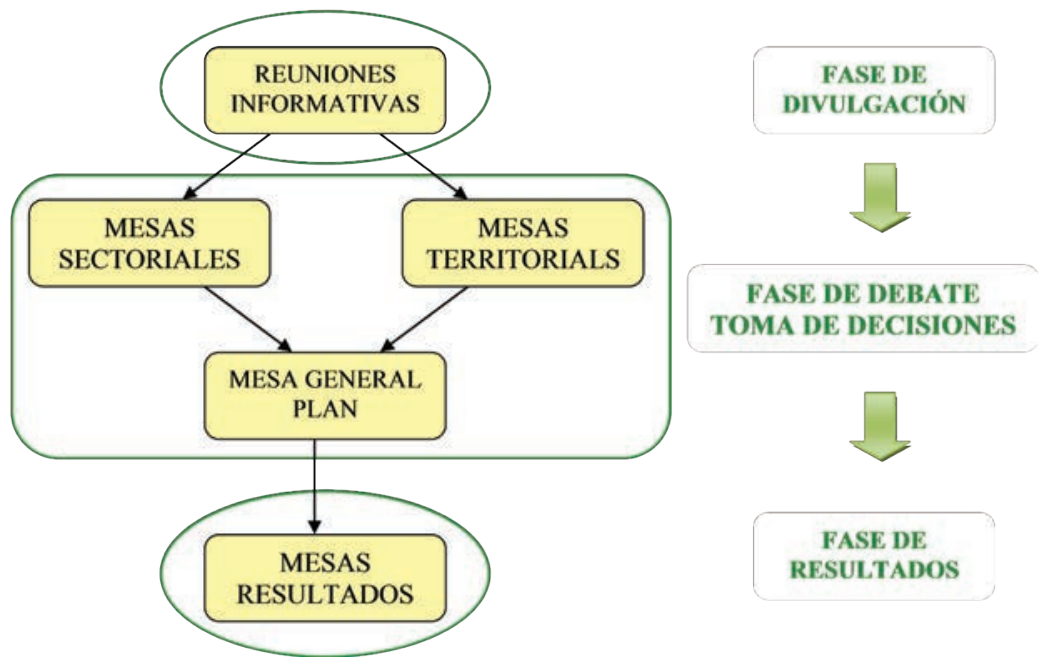

Figura 3. Esquema de participación pública desarrollado en el plan del distrito forestal de A Fonsagrada- Os Ancares.

Public participation process in the forest planning, A Fonsagrada- Os Ancares forestry district. 


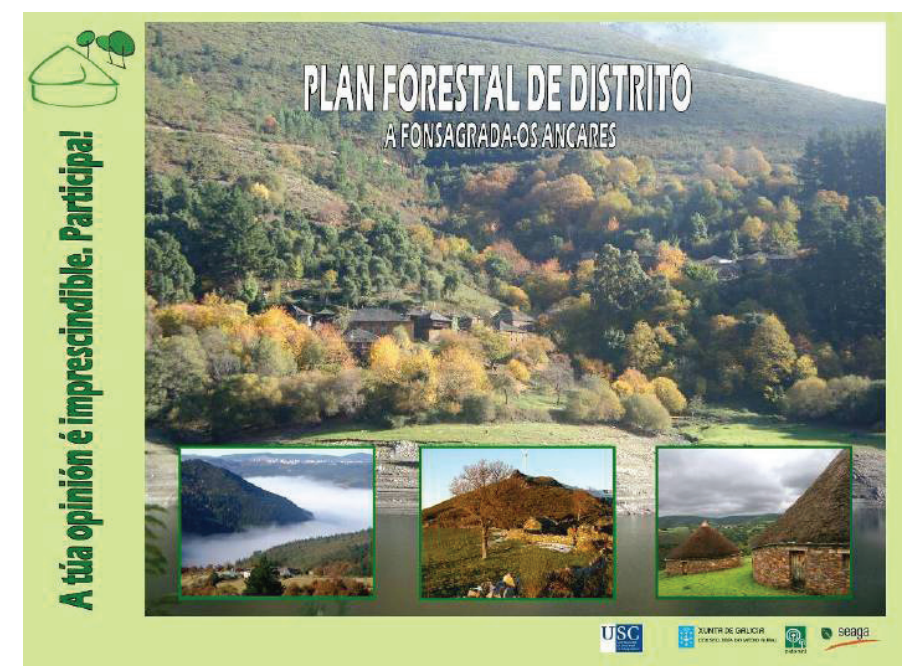

Figura 4. Modelo de cartel informativo para difusión del plan forestal, distrito forestal A Fonsagrada- Os Ancares. Model of informative poster for advertising the forest planning, A Fonsagrada- Os Ancares forestry district.

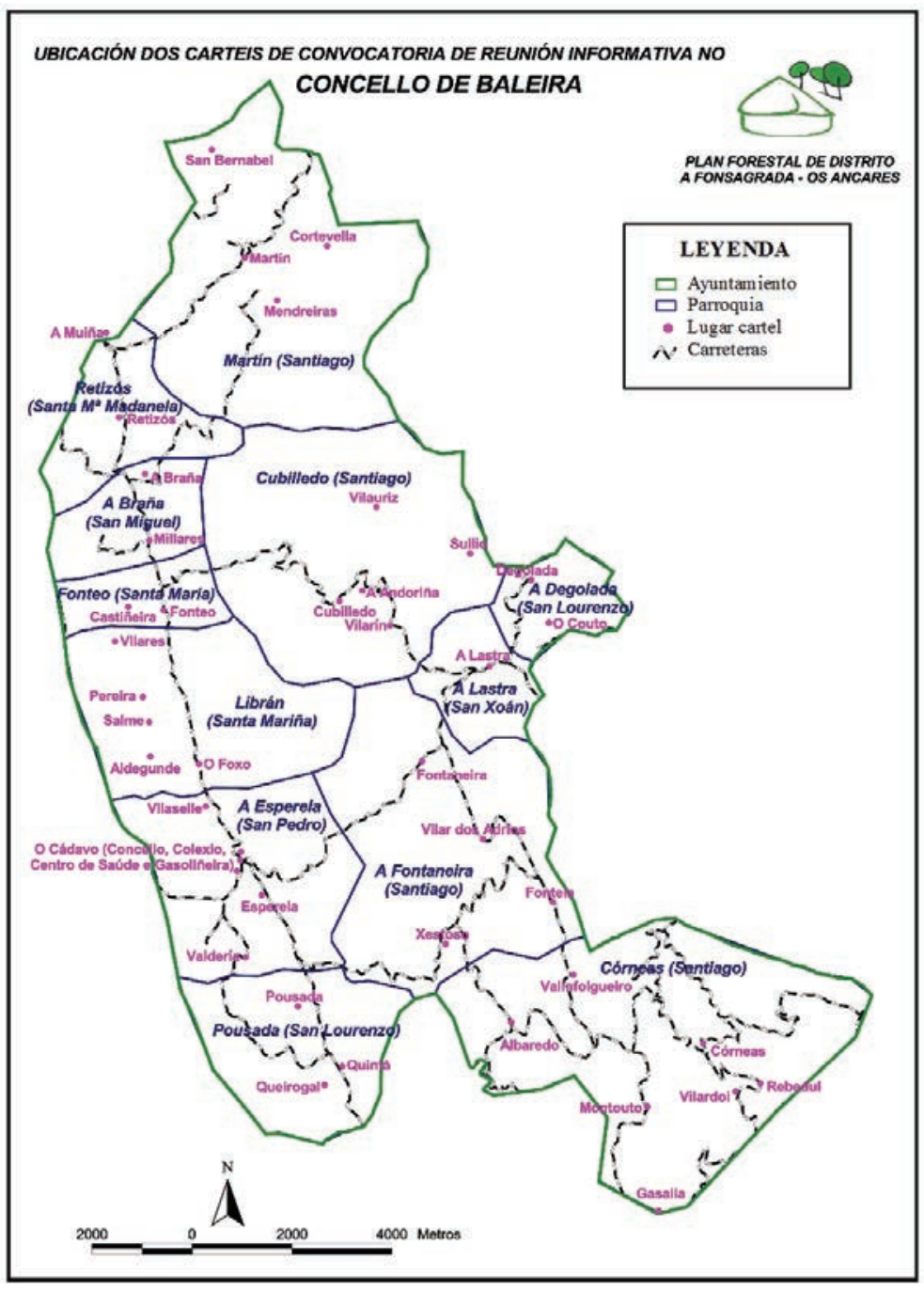

Figura 5. Distribución territorial de carteles informativos para difusión del plan forestal, distrito forestal A Fonsagrada- Os Ancares. Territorial distribution of informative posters for advertising the forest planning, A Fonsagrada- Os Ancares forestry district. 
Fase debate y toma decisiones. Esta fase se estructuró mediante la constitución de dos tipos de mesas de trabajo: sectoriales y territoriales; ambas con un número ilimitado de participantes. Las mesas sectoriales estaban formadas por aquellos grupos de interés de la zona vinculados directamente con el ámbito forestal, distinguiendo tres grandes grupos: propietarios, usuarios de recursos forestales y reguladores. Por su parte, las mesas territoriales eran herramientas de debate creadas por agentes clave vinculados a territorios o zonas con características específicas dentro de la región de estudio, distinguiendo grupos en base al territorio al que perteneciesen dentro de la zona del plan forestal.

De los integrantes en las mesas descritas con anterioridad se seleccionaron los representantes para constituir las mesas del plan forestal de A Fonsagrada- Os Ancares, esto es, las mesas de aprobación y negociación de las cuestiones debatidas en las correspondientes mesas territoriales y sectoriales. Por operatividad, estas mesas contaron con un número estable de miembros, que se fijó entre los 10 y los 15 miembros, representando a todos los stakeholders y a ambos tipos de mesas de debate.

Los tres tipos de mesas fueron convocados para el debate y análisis de los niveles decisivos para la elaboración y redacción técnica del plan forestal del distrito: inicio, diagnóstico y análisis, planificación y objetivos.

Para trabajar de forma objetiva con las opiniones de todos los participantes de las distintas mesas y obtener así, de forma colectiva, una radiografía de la visión actual y futura de los recursos forestales de la región a planificar, se diseñó un total de nueve modelos de cuestionario, en base al colectivo que representaba cada tipo de participante (propietarios forestales, comuneros, apicultores, cazadores, ganaderos, grupos ecologistas, empresas forestales, personal de la administración pública y diversos colectivos con intereses en el sector forestal). El esquema general del cuestionario estaba formado por un total de 125 preguntas estructuradas en cuatro grandes bloques: perfil, opinión, problemas específicos y características de su actividad. Indicar que las 34 preguntas relacionadas con los dos bloques opinión y problemas específicos eran comunes para los nueve modelos de cuestionario, siendo cuestiones relativas a criterios e indicadores forestales pan-europeos (MCPFE 2000) con el objetivo de comparar los principales elementos de planificación forestal con otros territorios y períodos temporales.

Fase de resultados. Finalizadas las fases anteriores, se realizó una exposición pública con los resultados iniciales. La documentación generada por la redacción técnica del plan forestal, así como la planificación de los recursos forestales, se expuso en todas las oficinas públicas presentes en el distrito.

Además, se realizó una serie de exposiciones y charlas aclaratorias de los resultados para cada municipio del distrito forestal. Como apoyo final al proceso planificador, se recogieron las sugerencias de la población a la información expuesta para su debate a través de la mesa general del plan y su idoneidad para incorporar en el plan forestal. Se mantuvieron ocho reuniones presentando los resultados, a nivel sectorial y territorial, participando un total de 59 personas.

Las fases anteriores fueron publicitadas en el sitio web creado para el plan forestal, así como en los lugares públicos establecidos para informar en cada municipio y dependencias administrativas existentes.

\section{RESULTADOS}

Fase de divulgación. Considerando la experiencia y conocimiento detallados del entramado social de la zona de estudio por parte del equipo investigador, se identificaron tres grandes categorías de participantes clave para la planificación del distrito forestal A Fonsagrada- Os Ancares: propietario, regulador y usuario de recursos forestales. Dentro de estas categorías principales, se identificó un total de 12 grupos distintos de interés: propietarios forestales privados individuales, propietarios forestales privados colectivos o comuneros, administraciones locales, administración forestal, agricultores o ganaderos, cazadores, apicultores, empresas forestales, otras empresas relacionadas, colectivos ecologistas, colectivos sin ánimo de lucro y grupos de desarrollo local. Tanto el número de categorías (tres) como el número de grupos (doce) eran similares a las cifras medias de trabajo manejadas en otras experiencias de participación pública (Sheppard y Meitner 2005, Primmer y Kyllönen 2006, Hiltunen et al. 2009, Kangas et al. 2010).

El cuadro 1 refleja los datos de participación en las reuniones por municipio del distrito (nivel territorial), un total de 453 personas, es decir, el 2,37 \% de la población de la región. Por su parte, el cuadro 2 detalla los datos de inscripción en las reuniones informativas por stakeholder (nivel sectorial), un total de 303 personas, es decir, el 1,32 \% de la población inicial. Por tanto, más del 60 \% de los que asistieron a las charlas informativas se inscribieron finalmente en el proceso, claro indicador sobre el éxito que tuvieron dichas reuniones. En el cuadro 1 se aprecia cómo la mayoría de los asistentes a las reuniones se inscribieron en el proceso participativo del plan forestal.

Terminado el período para inscribirse, se inició la segunda parte del proceso, la constitución de mesas, clasificando a los participantes en base a su categoría y stakeholder al que representasen (cuadro 2).

Fase de debate y toma de decisiones. Para esta fase se tuvo que diseñar un cuestionario que recogiese la opinión y expectativas de numerosas personas y además, que aportase una radiografía colectiva de la actual consideración del ámbito forestal presente en la zona y de sus necesidades futuras. Para conseguir esto, se realizaron 134 cuestionarios individuales distribuidos por stakeholder, reflejado en el cuadro 3. Así, se podría analizar inicialmente la problemática existente entre los distintos stakeholders para, con posterioridad, consensuar y negociar vías posibles de solución en las mesas del plan. 
Cuadro 1. Cifras de asistencia e inscripción del proceso, por municipio, distrito forestal A Fonsagrada- Os Ancares. Attendance and enrollment in the public participation process, by municipality, A Fonsagrada- Os Ancares forestry district.

\begin{tabular}{lccccc}
\hline Municipio & $\mathrm{N}^{0}$ asistentes & $\mathrm{N}^{\circ}$ inscritos & \% inscripción & Población inicial & \% población inicial \\
\hline Baleira & 44 & 32 & 72,73 & 2.230 & 1,43 \\
Baralla & 48 & 23 & 47,92 & 2.546 & 0,90 \\
Becerreá & 72 & 44 & 61,11 & 3.406 & 1,29 \\
Cervantes & 71 & 54 & 76,06 & 2.837 & 1,90 \\
A Fonsagrada & 107 & 72 & 67,29 & 6.317 & 1,14 \\
Navia de Suarna & 57 & 18 & 31,58 & 1.866 & 0,96 \\
Negueira de Muñiz & 27 & 16 & 59,26 & 532 & 3,01 \\
As Nogais & 40 & 31 & 77,5 & 1.552 & 2,00 \\
Pedrafita do Cebreiro & 13 & 13 & 100 & 1.240 & 1,05 \\
\hline Total & 479 & 303 & 63,26 & 22.926 & 1,32 \\
\hline
\end{tabular}

Cuadro 2. Cifras de inscripción del proceso, por stakeholder, distrito forestal A Fonsagrada- Os Ancares.

Enrollment in the public participation process, by interest group, A Fonsagrada- Os Ancares forestry district.

\begin{tabular}{llrr}
\hline \multirow{2}{*}{ Categoría } & Grupo de interés & $\begin{array}{c}\mathrm{N}^{\circ} \\
\text { inscritos }\end{array}$ & \multicolumn{1}{c}{$\%$} \\
\hline \multirow{2}{*}{ Propiedad } & Propietarios individuales & 135 & 44,55 \\
& Comuneros & 81 & 26,73 \\
\hline \multirow{2}{*}{ Regulador } & Administraciones locales & 1 & 0,33 \\
& Administración forestal & 9 & 2,97 \\
\hline \multirow{4}{*}{ Usuarios } & Agricultores o ganaderos & 44 & 14,52 \\
& Cazadores & 8 & 2,64 \\
& Apicultores & 1 & 0,33 \\
& Otras empresas forestales & 10 & 3,30 \\
& Colectivos ecologistas & 1 & 0,33 \\
& Colectivos sin ánimo de lucro & 3 & 0,99 \\
& Grupos de desarrollo rural & 4 & 1,32 \\
\hline Total & & 303 & 100,00 \\
\hline
\end{tabular}

Como claro ejemplo de la información recogida en los cuestionarios, para su posterior trabajo de consenso en la toma de decisiones para la planificación forestal de la zona, se cita la pregunta, ¿está de acuerdo con la superficie forestal existente? Los resultados indicaron que un $68 \%$ de los participantes consideraban que la superficie forestal actual del distrito A Fonsagrada- Os Ancares era adecuada o suficiente, señalando indirectamente el desinterés por ampliar este tipo superficie en la zona (figura 6).
Otro ejemplo relevante fue la valoración de las formaciones paisajísticas presentes en el distrito por parte de los distintos grupos de interés participantes. Como refleja la figura 7 , el deseo generalizado de la población encuestada era tener un paisaje forestal diversificado en la región.

Ante estas y otras evidencias obtenidas, el plan forestal del distrito debía engranar estas preferencias sociales, planificando la diversificación de formaciones arbóreas de la región, pero sin aumentar su superficie forestal. Este tipo de decisiones representan claros ejemplos de cómo incorporar medidas operativas que generen consenso en la elaboración de un plan forestal.

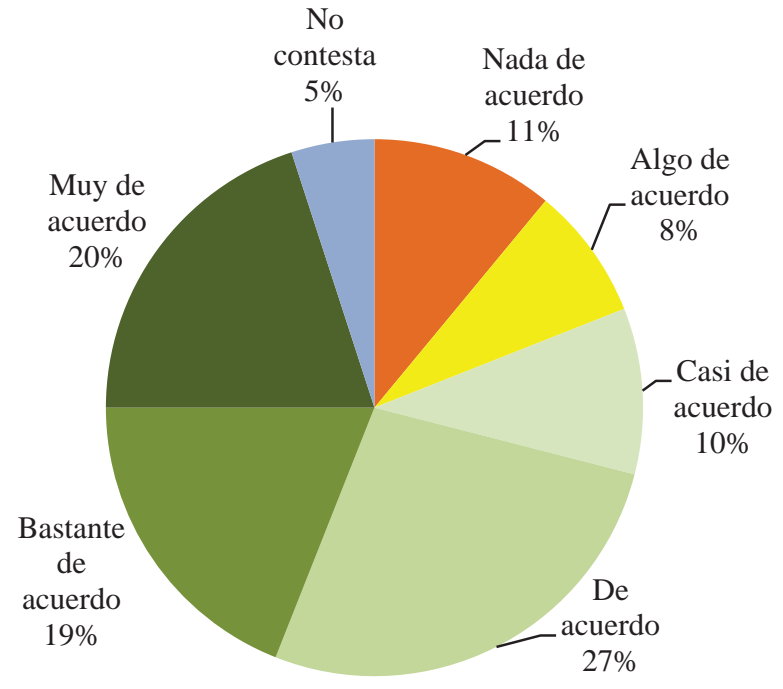

Figura 6. Representación gráfica de los resultados a la pregunta: ¿Está de acuerdo con la superficie forestal existente?

Graphic representation of the question results: Do you agree with the existing forest area? 
Cuadro 3. Número de encuestados, por municipio y grupo de interés, distrito forestal A Fonsagrada- Os Ancares. Respondents, by municipality and interest group, A Fonsagrada- Os Ancares forestry district.

\begin{tabular}{|c|c|c|c|c|c|c|c|c|c|c|}
\hline Municipio & Apicultor & Cazador & Comunero & Empresa & Ecologista & $\begin{array}{c}\text { Mesa } \\
\text { territorial }\end{array}$ & Administrativo & Propietario & Ganadero & Total \\
\hline Baleira & & 1 & 2 & & & & & 7 & 1 & 11 \\
\hline Baralla & & & 4 & & & & & 1 & & 5 \\
\hline Becerreá & & & 3 & 2 & & 1 & 3 & 9 & 4 & 23 \\
\hline Cervantes & & & 7 & 1 & & & & 9 & 1 & 18 \\
\hline A Fonsagrada & & 1 & 4 & & & & 1 & 8 & 3 & 17 \\
\hline Navia de Suarna & 1 & & 6 & & & 1 & & 4 & & 12 \\
\hline Negueria de Muñiz & & & 12 & & & 1 & & 2 & 1 & 16 \\
\hline As Nogais & & & 9 & & & 1 & & 8 & & 18 \\
\hline Pedrafita do Cebreiro & & & 3 & & & & & 1 & & 4 \\
\hline Lugo & & & 1 & & 2 & 2 & 2 & & & 7 \\
\hline Santiago & & & & 1 & & 1 & & & & 2 \\
\hline Madrid & & & & & 1 & & & & & 1 \\
\hline Desconocido & & & & & & & 1 & & & 1 \\
\hline Total & 1 & 2 & 51 & 4 & 3 & 7 & 7 & 49 & 10 & 134 \\
\hline
\end{tabular}

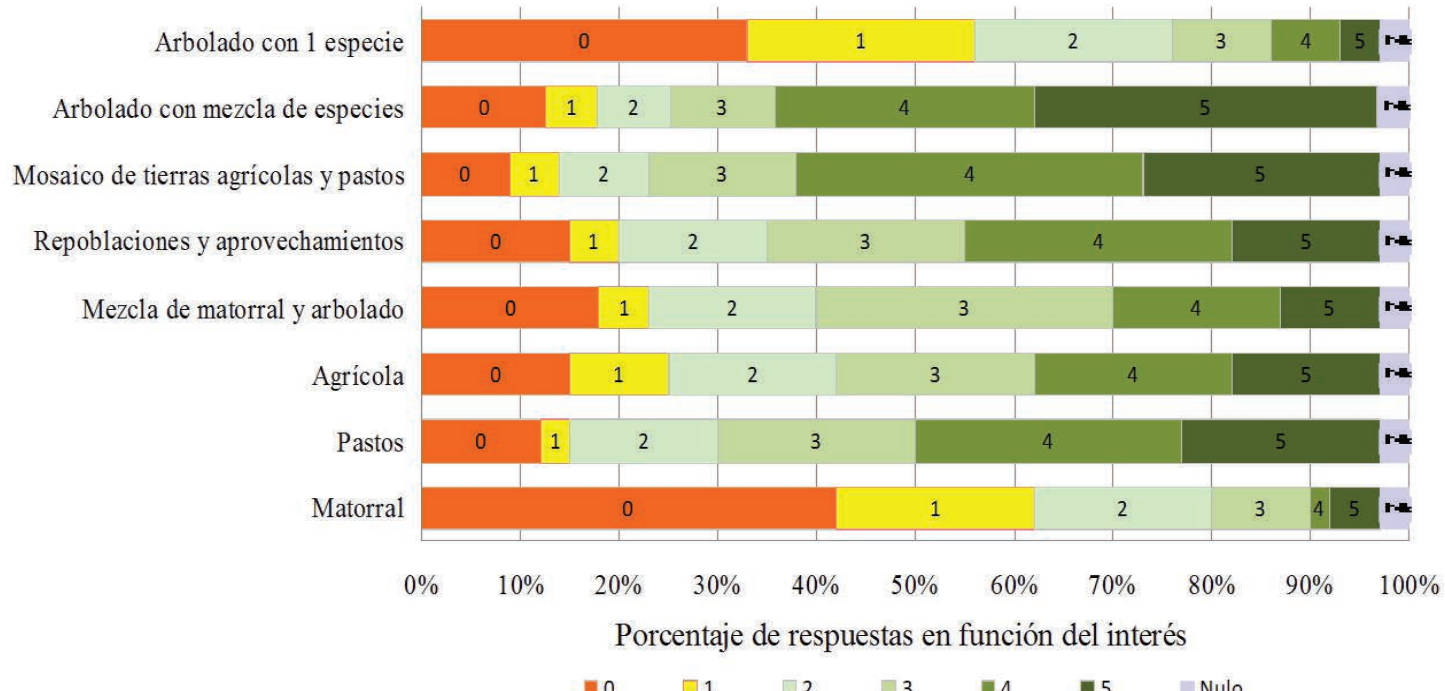

Figura 7. Representación gráfica de los resultados a la valoración de las formaciones paisajísticas de la zona (0 menor valor, 5 mayor valor).

Graphic representation of the value results about the landscape units of the area ( 0 minor value, 5 major value).

Fase de resultados. En esta última fase se validaron los resultados obtenidos en la etapa anterior, matizándose y concretándose las propuestas colectivas que se incluyeron dentro del plan forestal del distrito. Siguiendo con los ejemplos indicados previamente, en este punto se confirmó la decisión de planificar la superficie forestal de la zona mediante la diversificación de sus formaciones arbóreas, pero sin aumentar la superficie dedicada a este uso.
Finalmente, se cita que el número de personas participantes fue disminuyendo con el paso del tiempo (figura 8), trabajando en todas las fases el 19,5 \% de la población inscrita. Este porcentaje fue similar para cada municipio del distrito y para cada grupo de interés participante, destacando la presencia de todos los grupos de interés en cada una las fases. 


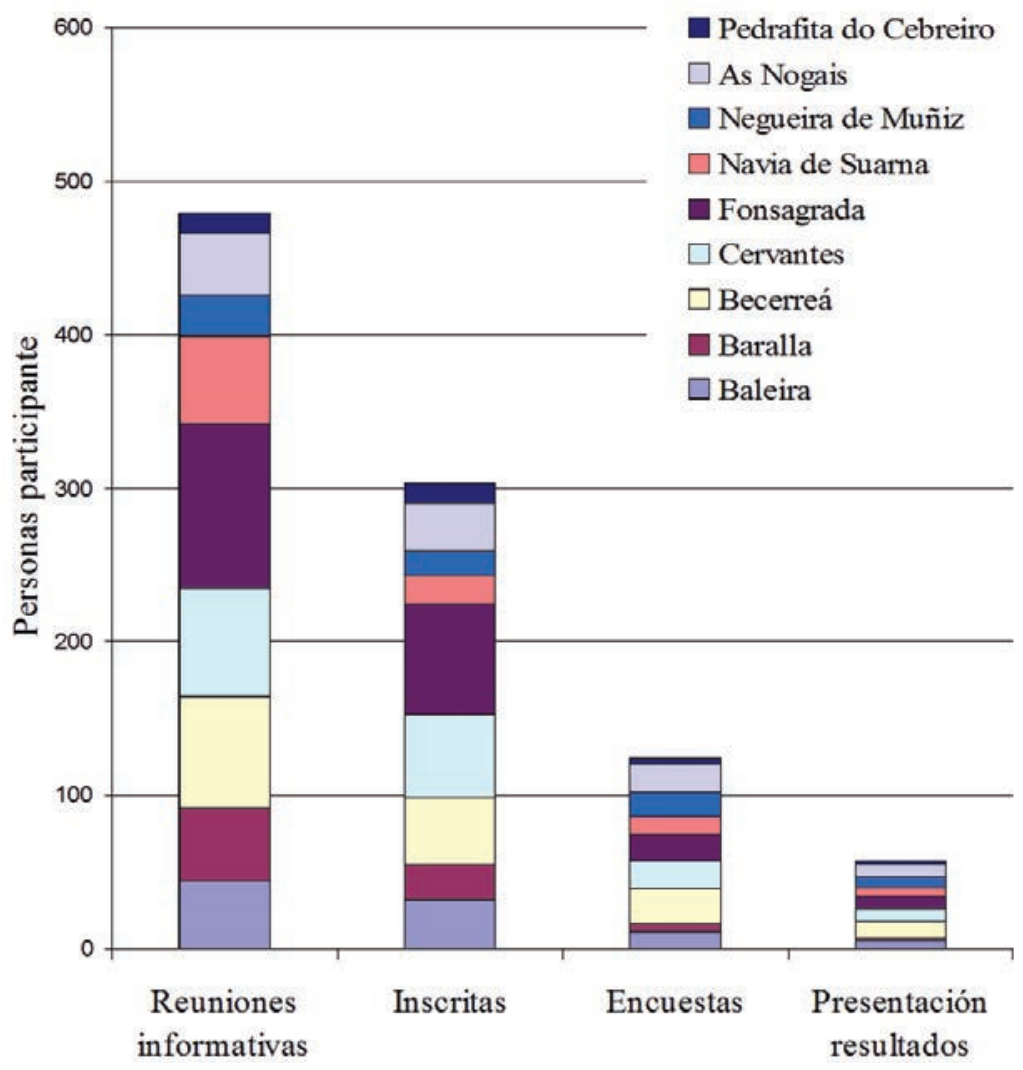

Figura 8. Evolución del número efectivos durante proceso de participación pública, distrito forestal A Fonsagrada- Os Ancares (año 2009).

Evolution of the participant number during public participation process, A Fonsagrada- Os Ancares forestry district (2009 year).

\section{DISCUSIÓN}

En todo proceso participado es necesario que se garanticen tres aspectos clave: equidad, representatividad y transparencia con la población (Buchy y Hoverman 2000). De hecho, la planificación participativa es una herramienta muy útil para evitar posibles conflictos sociales, compartir información y promover unas adecuadas relaciones entre la entidad promotora del plan y la población receptora (Hellström 2001, Hiltunen et al. 2009).

En concreto, desarrollar un plan forestal con participación pública es un claro reto para poder contribuir en todo proceso de democratización de diversas formas, ya sea reforzando la transparencia en los acuerdos políticos, ayudando en las decisiones más pluralistas, estimulando el conocimiento y comunicación mutuos, o concienciando sobre la responsabilidad colectiva en materia de gestión y planificación forestal (Kangas et al. 2010). En esta línea, la metodología del presente estudio permitió elaborar, de forma participativa, el plan forestal de una determinada región rural sin referentes previos en participación social, consiguiendo romper la continuidad de planificaciones dirigidas y verticales, tradicionalmente propias del campo forestal que han demostrado su fracaso en más de medio siglo de aplicación.
De hecho, la participación pública en material forestal es constantemente reconocida como uno de los elementos llave en la planificación forestal, en base a la gestión de conflictos, el acercamiento al consenso, la colaboración en solucionar problemas y la negociación para tomar decisiones (Bruña-García y Marey-Pérez 2014). Por lo tanto, la utilización de participación pública dentro del plan forestal del distrito A Fonsagrada- Os Ancares ha permitido conocer la existencia de conflictos y encauzar posibles soluciones de consenso para el futuro del sector forestal de la región.

No obstante, los responsables técnicos y políticos en materia de gestión y planificación forestal suelen mostrarse críticos con la aplicación de instrumentos participativos, basando su no aceptación en la orientación cualitativa de los resultados y de su falta de rigor y/o estructura de dichos enfoques. Así, la participación en el ámbito forestal ha sido definida como un mero proceso para identificar e interpretar la información aportada por los stakeholders (Bruña-García y Marey-Pérez 2014). Ante lo expuesto, la metodología utilizada en este estudio ha conseguido universalizar y sistematizar los resultados, manteniendo los elementos locales propios de la región.

Para la zona de estudio, la expectativa de no aumentar la superficie forestal, reflejada en los resultados obtenidos en los cuestionarios, se contrapone a la planificación tra- 
dicional forestal llevada a cabo hasta el momento (MareyPérez y Rodríguez-Vicente 2011); así, el principal interés de la administración pública forestal ha sido incrementar la superficie arbolada mediante la repoblación de terrenos de pasto o matorral en contra de la opinión generalizada de la población local, generando numerosos conflictos socioeconómicos (Gómez-Vázquez et al. 2009). Además, el interés de la población por diversificar el mosaico forestal de la región, reflejado también en los cuestionarios realizados, es contrario a la actividad repobladora con plantaciones monoespecíficas de pino incentivada desde la propia administración pública forestal cuyo paisaje resultante no ha sido inicialmente considerado como criterio de decisión (Jaraíz-Cabanillas et al. 2013).

Así, los resultados confirman la preferencia de la población de estudio por paisajes forestales que difieren de las prácticas clásicas de ordenación forestal, al igual que la población rural de otras regiones forestales (Nielsen et al. 2007). Por lo tanto, la planificación forestal esperada por los habitantes del distrito no coincide con la existente en la actualidad, siendo muy heterogéneo el tipo de paisaje diversificado que la población estima adecuado, si bien la tendencia principal es valorar más aquel paisaje que recuerda al paisaje tradicional de la zona (Díaz-Varela et al. 2009) o aquel paisaje que combina espacios naturales con superficies agrarias. Además, el interés social insiste no solo en diversificar la estructura, sino también en la composición de especies, suponiendo un elemento clave en el diseño de la gestión y planificación forestal futuras de la zona. Autores como Gundersen y Frivold (2008), mediante el empleo de cuestionarios sociales en zonas rurales nórdicas, obtuvieron resultados similares a los del presente estudio.

Finalmente se cita que uno de los principales obstáculos a la integración de procesos de participación pública en planificación forestal, un importante coste, tanto en tiempo como en aportación económica (Stenseke 2009), no supuso una traba para la validación de la metodología propuesta en la zona de estudio. En esta región, sin experiencia previa en participación pública, las diferentes etapas del proceso participativo supusieron un esfuerzo relativamente bajo, menos de 0,0033 horas de personal técnico por unidad de superficie (ha), es decir, menos de un año. Se observa, sin embargo, una disminución en el número de participantes a lo largo del proceso, hecho posiblemente asociado a la falta de cultura en materia de participación y compromiso por parte de los participantes, así como a posibles causas vinculadas al propio diseño del proceso: tiempo (se desarrolló durante casi un año), formato (demasiado formal y académico para una media de población con muchos años), estructura (que en las fases finales concentraba las reuniones en un único punto), etc.

\section{CONCLUSIONES}

El modelo propuesto, como parte integrante de la planificación forestal de un área rural, permite obtener infor- mación trascendente de todos los aspectos que inciden y caracterizan la planificación y gestión forestal pasadas, futuras y actuales de la zona. Con base en lo anterior, el modelo presentado clarifica los conflictos existentes en la región en materia forestal y permite encauzar vías para el consenso de la futura gestión de sus recursos forestales. Así, este proceso sienta las bases de la futura gobernanza rural de la región donde se desarrolla el proceso participativo.

La validación del proceso de participación pública, integrado en el plan forestal del distrito A Fonsagrada- Os Ancares, puede considerarse exitosa, gracias a la representación equilibrada alcanzada, tanto a nivel territorial como sectorial, en un período relativamente corto de tiempo (inferior a un año).

Desde una perspectiva preliminar, se podría afirmar que la mayoría de los conflictos para gestionar los recursos rurales de la zona, especialmente en materia forestal y de desarrollo rural, pueden comenzar a solucionarse con una medida muy sencilla: hablando con las personas interesadas en los recursos rurales del territorio. Esta medida no consume importantes medios económicos y de tiempo, si bien es más lenta que otros medios en su aplicación, permitiendo alcanzar un desarrollo más duradero y harmónico de la región y su población.

El éxito de toda medida a proponer en planificación forestal estará condicionado al grado de aceptación por parte de las personas clave en su realización futura y que, en el modelo propuesto, se identificaron con los grupos de interés previamente perfilados en la zona. Por tanto, alcanzar buenos resultados de gobernanza rural no sólo requeriría incorporar todos los grupos sociales clave en el proceso de planificación, sino que sería imprescindible un importante y continuo trabajo de extensión forestal y de intercambio social.

\section{AGRADECIMIENTOS}

A todas las personas que, con su participación, colaboraron activamente en la elaboración del plan forestal del Distrito A Fonsagrada- Os Ancares.

\section{REFERENCIAS}

Abelleira-Yáñez L, X Bruña-García, MF Marey-Pérez. 2011. El proceso de difusión en el proceso de participación pública para la elaboración de un PORF. Spanish Journal of Rural Development II (3): 63-74.

Álvarez-López CJ, JA Riveiro-Valiño, MF Marey-Pérez. 2008. Typology, classification and characterization of farms for agricultural production planning. Spanish Journal of Agricultural Research 6: 125-136.

Bruña-García X, MF Marey-Pérez. 2014. Public participation: a need of forest planning. iForest, Biogeosciences and Forestry. DOI: 10.3832/ifor0979-007

Buchy M, S Hoverman. 2000. Understanding public participation in forest planning: a review. Forest Policy and Econo- 
mics 1: 15-25.

Díaz-Varela ER, MF Marey-Pérez, A Rigueiro-Rodríguez, P Álvarez-Álvarez. 2009. Landscape metrics for characterization of forest landscapes in a sustainable management framework: potential application and prevention of misuse. Annals of Forest Science 66: 301-311.

Fuentes-Santos I, MF Marey-Pérez, W González-Manteiga. 2013. Forest fire spatial pattern analysis in Galicia (NW Spain). Journal of Environmental Management 128: 30-42.

Gómez-Vázquez I, P Álvarez-Álvarez, MF Marey-Pérez. 2009. Conflicts as enhancers or barriers to the management of privately owned common land: a method to analyze the role of conflicts on a regional basis. Forest Policy and Economics 11 (8): 617-627.

Gritten D, O Saastamoinen, S Sajama. 2009. Ethical analysis: a structured approach to facilitate the resolution of forest conflicts. Forest Policy and Economics 11 (8): 555-560.

Gundersen V, LH Frivold. 2008. Public preferences for forest structures: a review of quantitative surveys from Finland, Norway and Sweden. Urban Forestry \& Urban Greening 7: 241-258.

Hellström E. 2001. Conflict cultures- qualitative comparative analysis of environmental conflicts in forestry. Silva Fennica Monographs 2. Tampere, Finland. The Finnish Society of Forest Science. 109 p.

Hiltunen V, M Kurttila, P Leskinen, K Pasanen, J Pykäläinen. 2009. Mesta: An internetbased decision-support application for participatory strategic-level natural resources planning. Forest Policy and Economics 11(8): 1-9.

Higgs G, R Berry, D Kidner, M Langford. 2008. Using IT approaches to promote public participation in renewable energy planning: prospects and challenges. Land Use Policy 25: 596-607.

ILO (International Labour Office, IT). 2000. Public participation in forestry in Europe and North America. Report of the team of specialists on participation in forestry. WP 163. Geneva, Italy. FAO/ECE/ILO. 130 p.

Kangas A, N Saarinen, H Saarikoski, LA Leskinen, T Hujala, J Tikkanen. 2010. Stakeholder perspectives about proper participation for Regional Forest Programmes in Finland forest. Policy and Economics 12: 213-222.

Leskinen LA. 2004. Purposes and challenges of public participation in regional and local forestry in Finland. Forest Policy and Economics 6: 605-618.

Marey-Pérez MF. 2009. Plan Forestal del Distrito Fonsagrada os Ancares. Technical Project. España. Consellería de Medio Rural, Xunta de Galicia. 358 p.

Marey-Pérez MF, V Rodríguez-Vicente. 2011. Factors determining forest management by farmers in Northwest Spain: application of discriminant analysis. Forest Policy and Economics 13: 318-327.

MCPFE (Ministerial Conference on the Protection of Forests in Europe, ES). 2000. General Declarations and Resolutions adopted at the Ministerial Conferences on the Protection of Forests in Europe, Strasbourg 1990- Helsinki 1993- Lisbon 1998. Vienna, Austria. MCPFE Liaison Unit.

Nielsen AB, SB Olsen, T Lundhede. 2007. An economic valuation of the recreational benefits associated with naturebased forest management practices. Landscape and Urban Planning 80: 63-71.

Primmer E, S Kyllönen. 2006. Goals for public participation implied by sustainable development, and the preparatory process of the Finnish National Forest Programme. Forestry Policy and Economics 8: 838-853.

Pykäläinen J, V Hiltunen, P Leskinen. 2007. Complementary use of voting methods and interactive utility analysis in participatory strategic forest planning: experiences gained from western Finland. Canadian Journal of Forest Research 37(5): 853-865.

Rodríguez-Vicente V, MF Marey-Pérez. 2010. Analysis of individual private forestry in northern Spain according to economic factors related to management. Journal of Forest Economics 16: 269-295.

Utton A, WRD Sewell, T O’Riordan (eds). 1976. Natural resources for a democratic society: public participation in decision-making. Boulder, CO, USA. Westview Press. 236 p.

Sheppard SRJ, M Meitner. 2005. Using multi-criteria analysis and visualisation for sustainable forest management planning with stakeholder groups. Forest Ecology and Management 207: 171-187.

Stenseke M. 2009. Local participation in cultural landscape maintenance: lessons from Sweden. Land Use Policy 26: 214-223.

Sugimura K, TE Howard. 2008. Incorporating social factors to improve the Japanese forest zoning process. Forest Policy and Economics 10: 161-173. 
